\title{
X-ray analysis of disodium cromoglycate ${ }^{*}$
}

\author{
S. HAMODRAKAS, A. J. GEDDES AND B. SHELDRICK \\ Astbury Department of Biophysics, University of Leeds, Leeds LS2 9JT, U.K. \\ $X$-ray crystallographic studies have been made on the anti-asthmatic \\ drug disodium cromoglycate. Interesting features of the structure \\ are the non-coplanarity of the chromone (benzopyrone) rings, an \\ apparent disorder in the position of one of the sodium atoms, and the \\ occurrence of water-filled 'channels' in the crystal lattice.
}

Disodium cromoglycate (I; DSCG) was introduced in 1968 for the treatment of bronchial asthma. Evidence concerning its principal effects and possible mode of action has been described at some length in the recent literature (Cox, 1971; Cox \& Altoanyan, 1970). Crystals of the compound exhibit a remarkable reversible water absorption (Cox, Woodard \& McCrone, 1971), the number of water molecules per molecule of DSCG increasing from 5 or 6 at 30-70\% relative humidity (R.H.) to 9 at $90 \%$ R.H. Above $93 \%$ R.H. the crystals collapse to form a liquid crystal mesophase containing up to about 260 molecules of water per molecule of DSCG. The purpose of the present study is to determine the major structural features of the molecule in the crystal in the hope that this information will be valuable in future investigations into structure/activity relations and will help also in analysis of the transition from the crystalline to the liquid crystalline phase.<smiles>CC(O)COc1cccc2oc(C(N)=O)cc(=O)c12</smiles>

Crystals of DSCG were provided by Fisons Ltd., Loughborough, England. The space group was determined as P1 and the unit cell dimensions, obtained from Weissenberg photographs, were: $a=0.392, b=1.108, c=1.544 \mathrm{~nm}$ and $\alpha=$ $92.05^{\circ}, \beta=94.31^{\circ}, \gamma=94.36^{\circ}$. The measured density of $1.58 \mathrm{~g} \mathrm{cc}^{-1}$ indicated one molecule of DSCG and 5 or 6 molecules of water per unit cell. A set of threedimensional intensity data was obtained from equi-inclination Weissenberg photographs using the multiple film technique and visual estimation of the intensities. The structure was solved by a combination of Patterson and direct methods, using MULTAN (Germain, Main \& Woolfson, 1971) for the latter, and refined to a residual (R) value of $0 \cdot 152$. It was thought initially that the relatively high value of the $R$ factor might be due to low quality of the measured data, but a further partial data collection using a computer-controlled Enraf-Nonius CAD-4 four-circle diffractometer showed slight random fluctuations in the unit cell dimensions with corresponding small changes in the measured intensities. It is thought likely that these changes are caused by the reversible absorption of molecules of water (cf. Cox \& others, 1971).

A projection of the molecule down the a-axis is shown in II. Bond lengths, bond angles, and torsional angles are given in Tables 1, 2, and 3 respectively, with the atom

\footnotetext{
* Intal: disodium salt of 1,3-bis(2-carboxychromon-5-yloxy)-2-hydroxypropane (shown in I). Also known as Cromolyn Sodium or Lomudal.
} 
Table 1. Bond lengths $(\AA)$ and estimated standard deviations.

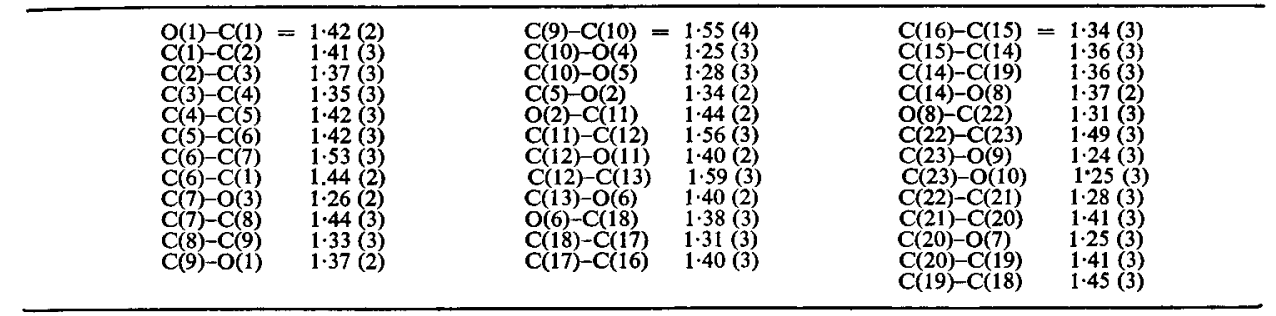

numbers corresponding to those of II. The planes containing the chromone rings are inclined to each other at about $53^{\circ}$, a feature which was readily discernible in the Patterson synthesis. This contained strong peaks in two distinct planar hexagonal nets at about this inclination. As expected, the carboxyl groups are almost co-planar with the chromone ring systems. The approximate planarity of the $\mathrm{C}(4)-\mathrm{C}(5)-0(2)-$ $\mathrm{C}(11)$ and $\mathrm{C}(17)-\mathrm{C}(18)-\mathrm{O}(6)-\mathrm{C}(13)$ groups and the short lengths of the $\mathrm{C}(5)-\mathrm{O}(2)$ and $\mathrm{C}(18)-\mathrm{O}(6)$ bonds $(0.1339$ and $0.1379 \mathrm{~nm}$ respectively) suggest a partial double bond character of these bonds. Another interesting feature of the compound is the way the $\mathrm{C}(12)-\mathrm{O}(11)$ bond in the bridge projects away from the planes of the ring systems.

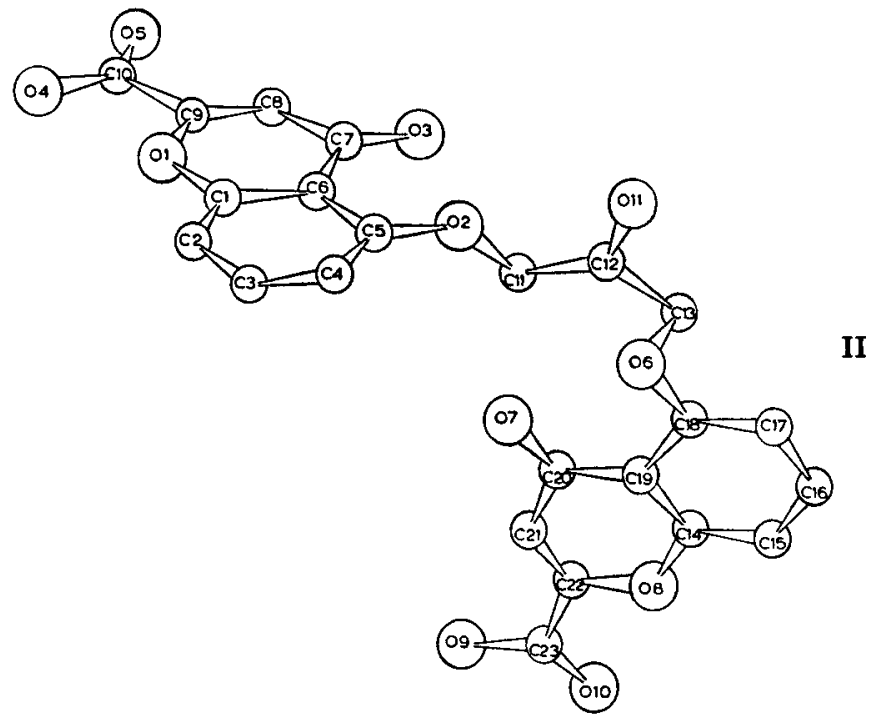

Table 2. Bond angles and estimated standard deviations.

\begin{tabular}{|c|c|c|c|c|c|}
\hline $\begin{array}{l}C(6)-C(1)-O(1) \\
C(6)-C(1)-C(2) \\
O(1)-C(1)-C(2) \\
C(1)-C(2)-C(3) \\
C(2)-C(3)-C(4) \\
C(3)-C(4)-C(5) \\
C(4)-C(5)-C(6) \\
C(4)-C(5)-O(2) \\
C(6)-C(5)-O(2) \\
C(5)-C(6)-C(1) \\
C(5)-C(6)-C(7) \\
C(1)-C(6)-C(7) \\
C(6)-C(7)-C(8) \\
C(6)-C(7)-O(3) \\
C(8)-C(7)-O(3) \\
C(7)-C(8)-C(9) \\
C(8)-C(9)-O(1) \\
C(8)-C(9)-C(10)\end{array}$ & $\begin{array}{c}=119.5(1.6)^{\circ} \\
122.6(1.8) \\
117.9(1.4) \\
119.6(1.7) \\
118.8(1.8) \\
124.4(1.8) \\
118.5(1.6) \\
128.0(1.6) \\
113.5(1.5) \\
115.8(1.8) \\
125.8(1.5) \\
118.3(1.7) \\
116.2(1.5) \\
124.5(1.6) \\
119.2(1.7) \\
120.5(1.9) \\
125.4(1.8) \\
121.0(1.9)\end{array}$ & $\begin{array}{l}C(10)-C(9)-O(1)= \\
C(9)-C(10)-O(4) \\
C(9)-C(10)-O(5) \\
O(4)-C(10)-O(5) \\
O(2)-C(11)-C(12) \\
C(11)-C(12)-O(11) \\
C(11)-C(12)-C(13) \\
O(11)-C(12)-C(13) \\
C(12)-C(13)-O(6) \\
C(15)-C(14)-C(19) \\
C(15)-C(14)-C(8) \\
O(8)-C(14)-C(19) \\
C(14)-C(15)-C(16) \\
C(15)-C(16)-C(17) \\
C(16)-C(17)-C(18) \\
C(17)-C(18)-O(6) \\
C(17)-C(18)-C(19) \\
O(6)-C(18)-C(19)\end{array}$ & $\begin{array}{l}113.7(1.6) \\
115.7(1.9) \\
117.9(1.8) \\
126.3(2.0) \\
102.1(1.5) \\
108.6(1.5) \\
108.6(1.5) \\
105.9(1.4) \\
99.9(2.4) \\
126.8(1.7) \\
112.7(1.6) \\
120.3(1.7) \\
117.6(1.8) \\
121.1(1.8) \\
118.7(1.7) \\
124.4(1.6) \\
124.0(1.8) \\
111.6(1.7)\end{array}$ & $\begin{array}{l}C(14)-C(19)-C(18) \\
C(14)-C(19)-C(20) \\
C(18)-C(19)-C(20) \\
C(19)-C(20)-O(7) \\
C(19)-C(20)-C(21) \\
O(7)-C(20)-C(21) \\
C(20)-C(21)-C(22) \\
C(21)-C(22)-O(8) \\
C(21)-C(22)-C(23) \\
O(8)-C(22)-C(23) \\
C(22)-C(23)-O(9) \\
C(22)-C(23)-O(10) \\
O(9)-C(23)-O(10) \\
C(1)-O(1)-C(9) \\
C(5)-O(2)-C(11) \\
C(13)-O(6)-C(18) \\
C(14)-O(8)-C(22)\end{array}$ & $\begin{array}{l}=111.5(1.8) \\
121.7(1.7) \\
126.7(1.8) \\
125.2(1.7) \\
113.3(1.8) \\
121.3(1.7) \\
122.3(1.9) \\
124.9(1.7) \\
122.5(1.8) \\
112.1(1.6) \\
117.1(1.8) \\
114.8(1.7) \\
127.8(1.8) \\
120.0(1.3) \\
113.8(1.4) \\
114.7(1.5) \\
117.2(1.5)\end{array}$ \\
\hline
\end{tabular}




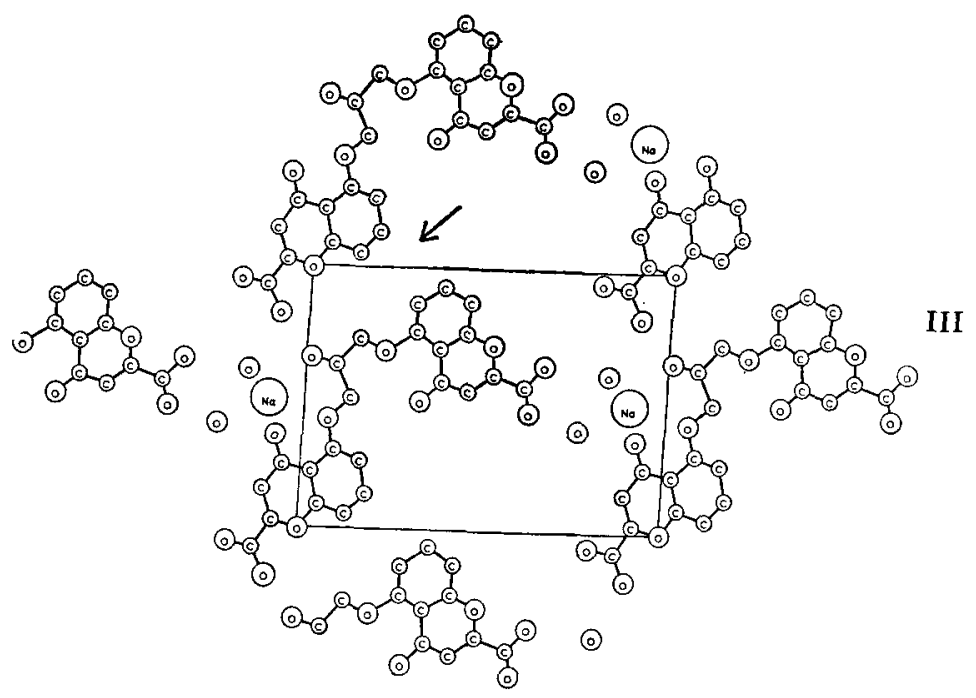

Table 3. Torsional angles.

\begin{tabular}{lrlr}
\hline$C(8)-C(9)-C(10)-O(5)=$ & $-4 \cdot 77^{\circ}$ & & \\
$C(4)-C(5)-O(2)-C(11)$ & 12.1 & $O(11)-C(12)-C(13)-O(6)$ & $175 \cdot 1$ \\
$C(5)-O(2)-C(11)-C(12)$ & $173 \cdot 3$ & $C(12)-C(13)-O(6)-C(18)$ & -178.4 \\
$O(2)-C(11)-C(12)-O(11)$ & $69 \cdot 0$ & $C(13)-O(6)-C(1)-C(17)$ & $-12 \cdot 2$ \\
\hline
\end{tabular}

Other aspects of the structure are best illustrated in the projection (III) along the a-axis showing the packing of adjacent molecules. Only one of the sodium atoms is clearly resolved and this is in a good position to coordinate with 7 oxygen atoms (4 from the cromoglycate and 3 from water molecules) two of which are shared with sodium ions in adjacent unit cells. There is a large space between the chromone rings sufficient to contain about 6 molecules of water, and, in three dimensions, this forms a large channel through the crystal lattice.

Most of these water molecules are discernible on the calculated electron density maps, but they have rather low peak heights indicating large thermal vibrations and/or a certain amount of disorder. Two of these peaks might also represent alternative positions of the second sodium ion. We intend to investigate the crystal under varying physical conditions in order to find an environment in which the diffraction is stable. This will enable us to collect more accurate data, in the hope of clarifying the positions of the molecules of hydration, and it would seem premature to discuss possible hydrogen-bonding systems etc. at this stage.

From an examination of X-ray powder photographs, Cox \& others (1971) report a significant change in the c-axis dimension only as the relative humidity is increased. This would be in accord with the proposed structure since there are no obvious forces holding the molecules together in this direction. In the b-direction there seems to be a region of 'hydrophobic' interactions (indicated with an arrow in III) while in the a-direction there are, of course, strong interactions between the stacked ring systems.

\section{REFERENCES}

Cox, J. S. G. (1971). Br. J. Dis. Chest, 65, 189-204.

Cox, J. S. G. \& Altoanyan, R. E. C. (1970). Respiration, 27 (suppl.), 292-309.

Cox, J. S. G., Woodard, G. D. \& McCrone, W. C. (1971). J. pharm. Sci., 60, 1458-1465.

Germain, G., Main, P. \& Woolfson, M. M. (1971). Acta Cryst., A27, 368-376. 